\title{
Optimization of Traffic Stream Using Simulation Tools
}

\author{
R.A. Filippov, L.B. Filippova*, A.S. Sazonova, A.A. Kuzmenko, E.V. Alekseeva \\ Bryansk State Technical University, Bryansk 241035, Russia \\ ${ }^{*}$ Corresponding author. Email: libv88@ mail.ru
}

\begin{abstract}
The article deals with the analysis and research of traffic stream intensity. The main points of developing and testing a simulation model of transport streams in AnyLogic are studied. An algorithm for conducting an optimization experiment is proposed and recommendations are made for optimizing traffic on the example of a single intersection in Bryansk. The proposed integrated approach allows to reduce transport and pedestrians' delays on busy roads due to the reconfiguration of traffic lights and optimization of public transport.
\end{abstract}

Keywords: simulation, transport stream simulation, optimization experiment, intersection

\section{INTRODUCTION}

The development of modern models and methods of logistics theory in organizing passenger transport services is aimed at improving the quality of life and forming "convenient logistics", implemented in introducing adaptive - smart - intelligent management systems in the city.

The research object of this paper is transport streams of the urban passenger transport system.

The research subject is the application of information technologies for optimizing transport logistics.

The purpose of the research is theoretical and methodological justification of using information systems for modeling and optimizing the urban passenger transport system.

The main problem of the urban transport system is the discrepancy between the capacity of the road network (RN) and the real demand for transport services [1]. The increase in traffic during rush hours, which exceeds the capacity of roads, is certainly the cause of congestions. It is possible to prevent traffic congestion by:

- $\quad$ increasing the capacity of roads: road expanding; building speed-change lanes; using intelligent transport systems; optimal traffic management;

regulation of road access: toll collection; restriction of entry; parking restriction.

The construction of new and reconstruction of existing road infrastructure facilities is the most obvious and cardinal solution to the problem of the city's RN congestion. To reduce the number of traffic jams, it is objectively necessary to reconstruct the busiest sections of highways and build bypasses of the most saturated urban areas in order to divert transit flows from them. At the same time, construction solutions are highly capital intensive and require considerable time [1].

The experience of many large megacities of the world shows that the construction of new and reconstruction of existing highways and roads with a constant increase in the number of vehicles does not allow to reduce completely the difference between RN capacity and the level of demand for road transport, since the introduction of a new highway section results in a sharp growth of traffic along it [1,5]. In a number of international cases, the problem of congestion of urban roads is solved by improving the efficiency of traffic management, including introduction and development of modern intelligent transport systems (ITS) that can provide traffic management on the existing $\mathrm{RN}$ without increasing the density of the road network [1]. One of the tools to make the urban passenger transport more attractive is effective transportation management, which is based on the use of advanced information technologies.

Traffic stream management refers to an area where conducting a full-scale experiment is difficult or impossible due to the fragility or cost of creating a prototype, the duration of the experiment in real time, etc. In this case, simulation is an effective tool for decision-making. Simulation is a research method in which a real system is replaced by a model that is being experimented with in order to obtain information about this system. One of the main advantages of this method is the ability to reproduce the system under study many times and determine its optimal state $[2,6,7]$. AnyLogic is used as a simulation modelling environment in the study.

\subsection{Problem Statement}

Simulation modeling allows to analyze traffic in various conditions, making it possible to take into account the separation of traffic lanes, individual and public transport, regulation by means of light signaling installations, etc. [2]. Traffic simulation can solve the following problems [3]: - evaluate the impact of the type of road intersection on traffic capacity;

- design, test and evaluate the impact of traffic light operation on the traffic;

- analyze traffic management on motorways, monitor traffic directions both on individual lanes and on the entire roadway; 
- analyze the capacity of large transport networks in the dynamic distribution of traffic streams;

- analyze the possibility of giving way to public transport;

- simulate planned road works and commissioning of new road sections;

- evaluate the transport efficiency of the proposed measures;

- simulate in details the movements of each participant;

- simulate public transport stops;

- calculate analytical indicators, make graphs of traffic stream indicators on a separate section of the simulated section, and on the road network as a whole. [1].

The urban passenger transport system of a large city is a complex system that includes a large number of interconnected and interacting components. Managing such a large system becomes more complex every year due to the growth of cities, the increasing number of vehicles, and changing needs of citizens in service. Domestic and foreign experience has proven the effectiveness of simulation modeling for making competent management decisions in the field of urban transport [3].

The issue of optimizing the route network of urban passenger transport is relevant for any city, and Bryansk is no exception. As the analysis of the state of the city's transport network and ways of organizing traffic shows, there are a number of problems that require finding scientifically based solutions. Since the organization of traffic management is an area where conducting a field experiment is difficult or impossible, simulation in many cases becomes the only tool for effective decision-making in this area. Experiments with real objects in this case are very difficult, will lead to delays in both private and public transport, inconvenience to passengers, and, consequently, to their dissatisfaction. One of the main advantages of this method is that, in contrast to analytical modelling simulation modelling of transport streams allows to reproduce the system under study many times and determine its optimal state [3].

\subsection{Peculiarities of Bryansk transport network}

At first glance, the transport network of Bryansk is overloaded with commercial vehicles of small passenger capacity, which are not related to the class of city buses. As a result, the following situation is observed in the system of urban passenger public transport in Bryansk:

- The city public transport consists of a bus and trolleybus fleet.

- Simultaneous service of various transport operators (commercial and municipal), each with its own class of road vehicles.

On the main routes passengers are transported by extralarge and large-capacity municipal buses, as well as on auxiliary routes by small-capacity buses.

- High traffic intensity of urban transport, associated with the duplication of routes and the use of small and especially small class vehicles.
- Non-compliance with traffic schedules.

- An increase in the number of commercial bus routes using a fleet of low-capacity buses has led to an outflow of passengers from municipal routes. This has resulted in unprofitable municipal transport, environmental degradation, congestion of the road network, and a significant reduction in the safety of passenger transport. In this regard, there are serious requirements for passenger transportation, its parameters and arrangement.

In Bryansk the situation on the roads is not so critical compared to the situation on the roads in Moscow. However, there are problems with congestion of roads in the central part of the city, especially during rush hours. The road network cannot cope with the daily traffic flow. Some of the most congested sections are ring roads, crossings and intersections. Some of the main places with large traffic jams are Lenin Avenue, Bezhitskaya Street, Krasnoarmeyskaya Street, etc. [1,2,4]

\section{SIMULATION MODEL CONSTRUCTION}

\subsection{Model algorithm of optimizing a road network section in order to reduce its congestion}

The intersection of Lenin Avenue and Fokina Street was selected for study and optimization in this study. A comprehensive approach was proposed to improve the transport situation of the object under study:

1 . Visual observation and analysis of indicators such as the length of a pedestrian crosswalk, the number of lanes in all directions, the number of controlled intersections, the frequency of vehicles in the aggregate and separately for minibuses, and other factors.

2. Creating a controlled intersection model in AnyLogic to describe the topology and main characteristics.

3. Transport survey of the intersection in order to obtain data on the actual movement of traffic flows.

4. Simulation of the traffic situation in AnyLogic in order to identify problem areas of the road network and make suggestions for their elimination.

5. Testing of the proposed solutions on a simulation model in order to identify their advantages and disadvantages.

6. Modernization of a specific node of the city's street and road network by implementing the best of the proposed solutions.

Based on the proposed comprehensive approach, an algorithm for optimizing a section of the road network was developed and implemented in order to reduce its congestion (Fig.1). 


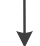

Initial data: traffic direction of vehicles at the intersection, the traffic

intensity in each direction including minibus taxis and buses, the

duration of each phase of the traffic lights, the vehicle's speed,

passenger traffic capacity and volume efficiency of minibus taxis

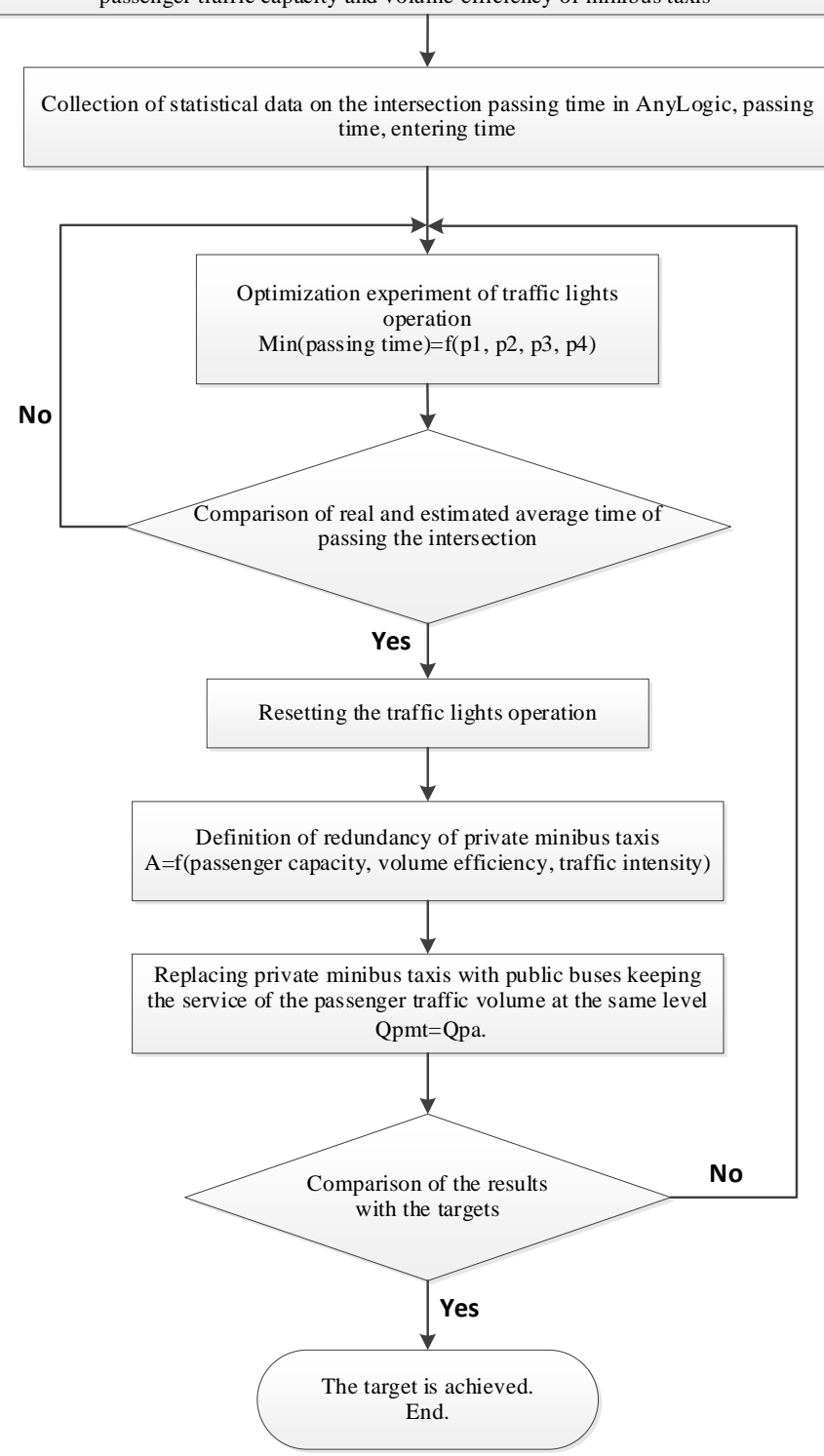

Figure 1 Model algorithm of optimizing a road network section in order to reduce its congestion

The algorithm describes the process of optimizing the congestion of a road network section by modeling in AnyLogic. The initial data for the algorithm are the parameters of road infrastructure, traffic intensity, and traffic light operation phases. To achieve the result, an optimization experiment is performed, the purpose of which is to achieve optimal traffic capacity parameters. Then, to achieve the greatest effect, the redundancy of minibus taxis is determined and an optimization proposal is made by replacing them with more passenger capable transport. If the optimization result is acceptable, i.e. reconfiguring the traffic light or replacing vehicles will have the effect of 
reducing downtime at key transport nodes (intersections, etc.), then the optimization will be completed and the algorithm is complete. Otherwise, you will need to go back to the new parameters of the optimization experiment and repeat the main steps with new conditions.

\subsection{Development of intersection simulation model in AnyLogic}

A simulation model of the intersection was built with all the surrounding roads, as well as a pedestrian crosswalk and traffic lights.

The topology of the road network in AnyLogic is set using the usual presentation shapes - lines and arcs.

The width of the shape (the thickness of its line) determines the number of lanes on the corresponding road section. The color of the road surface and road markings can be changed. The road network is set using the object roadNetwork, which checks whether the network is correct and displays the road network in the animation while the model is being made. This object must be present in any road traffic model. AnyLogic gives the possibility to create roads and vehicles as three-dimensional objects.

The developed simulation model of the intersection is based on the discrete event paradigm of simulation. During its construction, standard AnyLogic libraries were actively used, which provide fairly high functionality for modeling purposes.

Description of objects in the model (Fig. 2):

- road Network - this object sets the topology of the route transport network. The road network consists of 4 roads and 1 intersection.

- road Network Descriptor provides an access to control all vehicles located in the same road network. This block allows to set actions that will be performed when adding a vehicle to the road network, entering the road, vehicle stopping, changing lanes, and so on. In addition, one can use this block to display traffic jams on roads.

- car Source, bus Source, minibus Source are generators of cars, buses/ minibus taxis.

- car Move To, bus Move To, minibus Move To are blocks that control the movement of a vehicle (car, bus, or minibus, respectively). The target of the motion may be: a road, a bus stop or stop line. If a road is set as the target, you must also specify the direction of travel.

- car Dispose removes vehicles from the model.

- bus Stop is an object that simulates a stop point.

- ped Enter is the object that specifies the pedestrian's entrance to the transport network. Since the moment pedestrians enter, they begin to appear in the animation. Each pedestrian arriving at a stop has certain characteristics: a destination with $\mathrm{x}$ and $\mathrm{y}$ coordinates.

- ped Go To forces pedestrians to move to a specified location in the simulated space, which can be set by a line, point, or area. The transition will be considered completed when the pedestrian crosses the specified line, or reaches the specified point or area. Pedestrians will search for a path to the specified goal within the current floor.
- ped Sink removes pedestrians who entered the object from the simulated environment.

- ped Area Descriptor sets the area that defines the rules and/or imposes speed limits on pedestrians. It allows to change the speed of pedestrians and set their maximum speed.

- Delay is the object which simulates the idle time of the bus at a stop point.

- split is the object that creates a specified number of new copy agents for each incoming original agent.

$$
\begin{aligned}
& \checkmark \text { Main } \\
& >8 \text { Агенты } \\
& \checkmark \text { 祭 Презентация } \\
& \text { scale } \\
& \text { 䛗 image } \\
& \checkmark \text { roadNetwork } \\
& >\text { road } \\
& \text { 邦 intersection } 1 \\
& >\operatorname{road} 1 \\
& >\text { road } 3 \\
& >\text { road5 } \\
& \text { G. aвTo_presentation } \\
& \checkmark \text { ground } \\
& \square \text { wall } \\
& \checkmark \text { targetLine10 } \\
& \text { \ targetLine11 } \\
& \text { area } \\
& \text { 此 chart: Histogram Data ... }
\end{aligned}
$$

Figure 2 Simulation model tree

The number of created agents can change dynamically. The entire operation is performed within zero time - as soon as the agent enters the split object, it immediately leaves it along with the created agents.

Auto-population of cars is a set of agents of the same type. - traffic light is a traffic library element that can monitor traffic at specified intersections or stop lines.

The essence of the simulation experiment on the model is that vehicles of different types, with different speeds, move along roads at various time intervals.

The appearance of people at pedestrian crossings is associated with their getting off buses, then moving to the pedestrian crossing and crossing it diagonally, as the longest path.

Thus, during the experiment we can adjust the operation of traffic lights, add, remove or move pedestrian crossings, determine the degree of congestion of route network sections in the city, change the initial parameters (bus schedule, type and number of vehicles on the route, the routes themselves, the speed of buses, etc.), and based on the analysis of the situation, make decisions about the feasibility of these changes.

The initial data for making mathematical relationships were statistical information of the results of field observations.

Let's take a closer look at the structure and main components of the resulting model (Fig. 3).

A section of the road network and an intersection are based on a real satellite image uploaded to the program. The model scale is set according to the scale shown in the satellite image, that is 4 pixels correspond to $1 \mathrm{~m}$. 


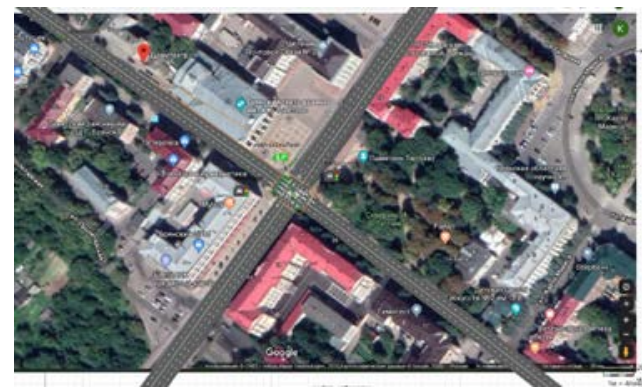

Figure 3 Model of road network section

\subsection{Model setting}

Since the intersection in question is complex due to the presence of additional traffic light sections, it is necessary to set the traffic light operation mode for the intersection lane connectors. This mode is used to control the traffic at an intersection where the lanes of the same road may have different phases (red / green). In this case, the traffic light mode should be set by specifying the color of the phases (red/yellow/green) and their duration in the Phase Table. Doing this, the lane connectors light up red/green, vehicles do not interfere with each other, and should not give way. This means that vehicles move correctly along the section of the road network.

When choosing a phase, one can see which directions are open for traffic during this phase. For the intersection under consideration, we set 7 traffic light phases, one of which will be red for all traffic directions and will correspond to the green light for pedestrians, and two will be yellow and will last 3 seconds. All traffic directions correspond to the actual traffic at the intersection under study.

To optimize the settings of the traffic lights, the parameters which will vary should be added. In this case, these are the duration of the traffic light phases, so four parameters will be added and default values will be specified for them that correspond to the actual values of the traffic lights. These options should be specified in the traffic light properties, namely in phase duration, respectively, the duration of the first phase will be set by $\mathrm{p} 1$ parameter, the duration of the second phase - p2 parameter, the third - p3, the fourth - p4. Now we make a new experiment - the optimization experiment. The average travel time of the intersection will be used as the target function.

Before starting the experiment, we will set the model time. In the training version, the time is limited to an hour of epy model operation, but this is quite enough, since several traffic light cycles will pass during this time. Then we launch the optimization model (Fig.4).

The graph shows the experiment procedure, the blue color indicates the optimal value at the moment, and it gradually improves.

Based on the results obtained, we can make a decision on setting real traffic lights to the optimal operating mode.

\begin{tabular}{l|r|r}
\multicolumn{2}{c}{$\begin{array}{c}\text { Crossroads: Optimization } \\
\text { Current status }\end{array}$} & \multicolumn{1}{c}{ Best } \\
\hline Iteration & 84 & 64 \\
Functional & 121.08 & 116.113 \\
\hline Parameters & & Copy best \\
\hline$p 1$ & 41 & 41 \\
\hline$p 2$ & 28 & 28 \\
\hline$p 3$ & 27 & 25 \\
\hline$p 4$ & 37 & 41 \\
\end{tabular}

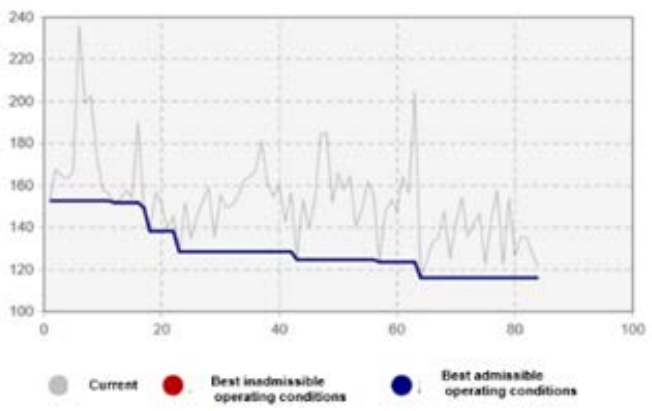

Figure 4 Search for optimal solution

\subsection{Optimization experiment results}

The degree of congestion of the city's route network sections on the model is determined by the color scheme (Fig. 5).

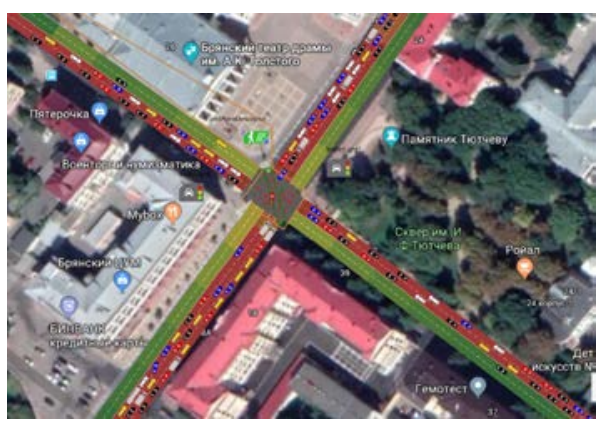

Figure 5 Traffic jams map on the route network section before optimization

Based on the above studies of passenger traffic on the routes passing along Lenin Avenue, we will try to reduce the number of minibuses by $10 \%$, and replace them with buses, taking into account their both passenger capacities.

Let us look at the traffic congestion after making changes in the simulation model (measurements were made at the same time after the experiment started) (Fig. 6). 


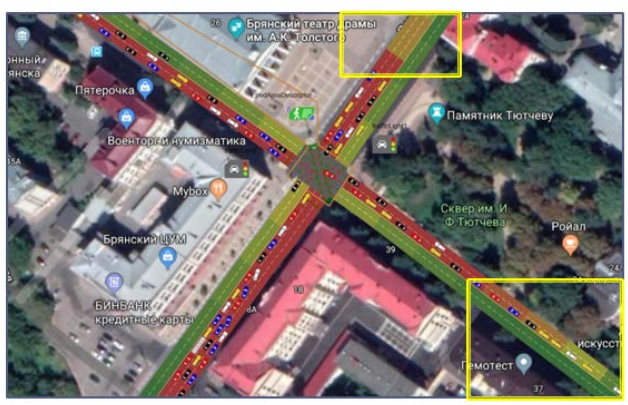

Figure 6 Traffic jams map on the route network section after optimization

The difference in traffic congestion before and after replacing taxis with buses can be judged by the color of the roads and the number of cars massed at the intersection in front of the stop lines (Fig. 7).

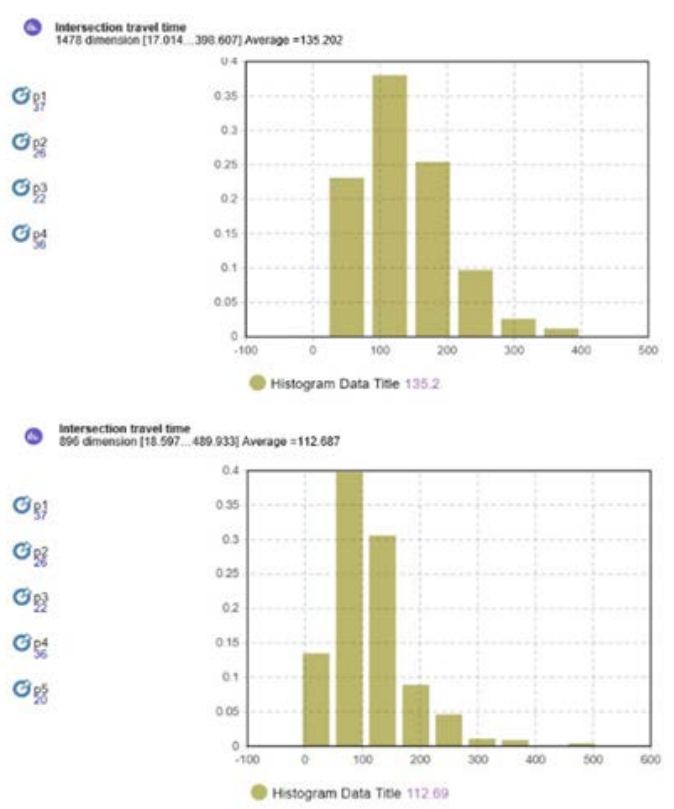

Figure 7 Average time value of intersection passing if minibuses are replaced with buses

In addition, the average time for vehicles to pass the intersection is now 112.687 seconds, which is $16.7 \%$ faster than when there are minibus taxis on roads.

\section{CONCLUSION}

The study proves the feasibility of using simulation programs to predict and optimize the system of urban passenger transport. The review of software tools is made and a comprehensive approach to the study of busy city sections is proposed. An algorithm for optimizing a section of the road network to reduce its congestion is developed. A simulation model of a real intersection is created.
A number of experiments are carried out on the developed simulation model and recommendations for optimizing a road network section are made.

\section{ACKNOWLEDGMENT}

This paper is written in Bryansk State Technical University at the department of Computer Technologies and Systems.

\section{REFERENCES}

[1] V.V. Sivakov, E.V. Alekseeva , Use of information technologies to improve the efficiency of passenger traffic accounting of urban transport, in: Ekonomika i effektivnost organizatsii proizvodstva, Bryansk State Engineering University of Technology, Bryansk 2018, no.27, pp. 108-110. [2] E.V. Alekseeva, V.V. Sivakov, Improving the efficiency of urban passenger transport. Innovatsionnoe razvitie i realizatsiya strategii formirovaniya tsifrovoy ekonomiki v Rossii. Proceedings of the International Conference. Novosibirsk State University of Architecture and Civil Engineering (Sibstrin). Novosibirsk, 2019, pp. 17-21.

[3] K.S. Borovaya, E.V. Alekseeva, Analysis of passenger traffic survey methods. Mashinostroenie: novie kontseptsii i tekhnologii. Proceedings of the International Scientific and Practical Conference of Students, Postgraduates and Young Scientists. Krasnoyarsk, Reshetnev Siberian State University of Science and Technology, 2019, pp. 82-86.

[4] F.Yu. Lozbinev, A.S. Sazonova, et al. Management in social and economic systems. Laboratory Course.

Moscow, 2019.

[5] Leonov E.A., Intellectual subsystems for collecting information from the internet to create knowledge bases for self-learning systems / E.A. Leonov, Y.A. Leonov, Y.M. Kazakov, L.B. Filippova/ In: Abraham A., Kovalev S., Tarassov V., Snasel V., Vasileva M., Sukhanov A. (eds) - Text : electronic // Proceedings of the Second International Scientific Conference "Intelligent Information Technologies for Industry” (IITI’17). IITI 2017. Advances in Intelligent Systems and Computing. 2017 - vol 679. — Springer, Cham, p. 95-103 DOI:10.1007/978-3-319-68321-8_10

[6] Sazonova A.S., Filippova L.B., et al. Calculation of a complex indicator of innovation potential, in: Kachestvo. Innovatsii. Obrazovanie, European Center for Quality, Moscow, 2018, no. 7, vol. 158, pp.5-9

[7] Leonov, YU.A. Selection of rational schemes automation based on working synthesis instruments for technological processes/ YU.A. Leonov, E.A. Leonov, A.A. Kuzmenko, A.A. Martynenko , E.E. Averchenkova, R.A. Filippov - Yelm, WA, USA: Science Book Publishing House LLC, 2019 - 192 p. — ISBN: 978-59765-4023-1 — Text : unmediated. 